\section{Nascimentos pré-termo no Brasil entre 1994 e 2005 conforme o Sistema de Informações sobre Nascidos Vivos (SINASC)}

\author{
Preterm births in Brazil from 1994 to 2005 \\ according to the Information System \\ on Live Births (SINASC)
}

\author{
${ }^{1}$ Faculdade de Medicina, \\ Universidade Federal de \\ Pelotas, Pelotas, Brasil. \\ 2 Programa de Pós-graduação \\ em Epidemiologia, \\ Universidade Federal de \\ Pelotas, Pelotas, Brasil. \\ 3 Secretaria de Vigilância em \\ Saúde, Ministério da Saúde, \\ Brasília, Brasil. \\ ${ }^{4}$ Escola de Enfermagem \\ Universidade Federal de \\ Minas Gerais, Belo Horizonte, \\ Brasil. \\ 5 Faculdade de Medicina, \\ Universidade de Brasília, \\ Brasília, Brasil. \\ Correspondência \\ M. F. Silveira \\ Departamento Materno- \\ Infantil, Faculdade de \\ Medicina, Universidade \\ Federal de Pelotas. \\ Av. Duque de Caxias 250, \\ Pelotas, RS \\ 96030-001, Brasil. \\ maris.sul@terra.com.br
}

\begin{abstract}
Monitoring preterm births is essential given their impact on infant morbidity and mortality and their economic and social costs. This article is based on data from the Information System on Live Births (SINASC), implemented in 1990 and expanded gradually to cover $90 \%$ of all births in the country. Preterm birth time trends are presented for Brazil, regions, and capitals from 1994 to 2005. At the national level, there was an increase in the preterm birth rate, accompanied by a reduction in the proportion of missing information on gestational age. The Southeast, South, and Central-West regions followed the national trend, while the preterm birth rate fell in the North and Northeast regions. We compared the findings from SINASC with those from population-based studies. The coverage and quality of SINASC has increased over time, but problems with the determination of gestational age still remain, leading to underestimation of preterm birth rates. Due to the importance of SINASC for monitoring, further efforts are needed to improve the system's accuracy.
\end{abstract}

Premature Infant; Information Systems; Health Planning

\author{
Mariângela F. Silveira 1,2 \\ Iná S. Santos 2 \\ Alicia Matijasevich 2 \\ Deborah Carvalho Malta 3,4 \\ Elisabeth Carmen Duarte 5
}

\section{Introdução}

No Brasil, vem se observando uma tendência de aumento da prematuridade. Estudos de coorte de nascimentos realizados em Ribeirão Preto (São Paulo) e Pelotas (Rio Grande do Sul) demonstraram claramente essa tendência 1. Em Ribeirão Preto, num período de 15 anos, a prevalência de prematuridade aumentou de $6 \%$ (1978 a 1979) para $13,3 \%$ (1994). Em Pelotas, o aumento foi de $6 \%$, em 1982, para 7,5\%, em 1993, e 15\%, em 2004 1. O aumento dos nascimentos pré-termo vem sendo evidenciado também em outros países. Nos Estados Unidos, entre 1992 e 2002, foi observada uma diminuição marcada de nascidos vivos com idade gestacional igual ou maior de 40 semanas e aumento daqueles com idade gestacional entre 34 e 39 semanas $(p<0,001)$, tanto em partos com ruptura prematura de membranas, quanto nos resultantes de intervenções médicas 2 .

A monitorização da prematuridade é muito importante, especialmente considerando seu impacto na morbidade e mortalidade infantis, além dos custos diretos do setor saúde associados ao manejo do nascido vivo prematuro. Os países desenvolvidos contam com sistemas complexos de coleta rotineira de dados demográficos, entre eles as características dos nascimentos ${ }^{3}$. Monitorar tendências e mudanças em indicadores de saúde, entre eles a prematuridade, ao longo do tempo, é essencial também para a avaliação do desempenho do sistema de saúde 4 . 
No Brasil, o Grupo de Estatísticas Vitais (GEVIMS) foi instituído através da Portaria $n^{\circ}$. 649/GM/MS, de 04 de julho de 1989, com o objetivo de criar um sistema de informação próprio sobre nascido vivo ${ }^{5}$. A partir do trabalho do GEVIMS, foi criado o Sistema de Informações sobre Nascidos Vivos (SINASC), adotando um documento-padrão nacional - a Declaração de Nascido Vivo (DNV) -, aprovado em janeiro de 19905.

O SINASC começou a ser implantado em 1990 e sua expansão para todas as Unidades da Federação aconteceu de forma gradativa. Desde o ano de 1994, o sistema é considerado implantado e apresenta, no âmbito nacional e em todas as regiões, uma cobertura maior de notificações de nascidos vivos do que aquela publicada pelo Instituto Brasileiro de Geografia e Estatística (IBGE) -, feita com base nos registros de nascidos vivos dos cartórios de registro civil 5,6. O sistema foi informatizado desde sua implantação, possibilitando a construção de indicadores úteis para o planejamento e gestão dos serviços de saúde, por meio da elaboração de relatórios de freqüência de eventos e de cruzamentos entre as variáveis 5. No Estado de São Paulo, o SINASC só foi considerado implantado a partir do ano 2000.

Este estudo visa a descrever a evolução da prematuridade no Brasil, regiões e capitais, entre os anos de 1994 e 2005, de acordo com dados do SINASC.

\section{Métodos}

Foram analisados os dados do SINASC gerenciados pelo Ministério da Saúde e disponíveis online na página do Departamento de Informática do SUS (DATASUS; http://tabnet.datasus.gov.br/ cgi/deftohtm.exe?sinasc/cnv/nvuf.def). O formulário da DNV contém várias informações, entre as quais foram analisadas a freqüência de nascidos vivos, segundo a duração da gestação em semanas, o local de residência da mãe e o local do parto (se hospitalar ou não).

Para os dados sobre o percentual de partos hospitalares no Brasil, no mesmo período, utilizou-se a informação disponível na página do DATASUS (http://www.datasus.gov.br/idb). Esse percentual é calculado com base no número de partos com informação sobre o local de ocorrência destes.

As proporções de prematuridade para todas as regiões do país e capitais estaduais foram calculadas ano a ano, sendo a tendência temporal apresentada na forma de gráficos. Os indicadores das capitais foram também analisados separadamente, por estas apresentarem maior homoge- neidade na qualidade da informação do que as demais cidades de cada estado.

\section{Resultados}

O percentual de nascimentos pré-termo $(<37$ semanas) no Brasil variou de 5\%, em 1994, a 6,6\%, em 2005, sugerindo um aumento na proporção de partos prematuros no país como um todo neste período ( $\mathrm{p}<0,001)$. Em 1994, o grupo de idade entre 28 e 36 semanas era responsável por $85,5 \%$ dos casos de prematuridade e, em 2005, o percentual foi de 94,6\% ( $p<0,001)$. A partir de 2001, tornaram-se disponíveis para todo o Brasil dados sobre o número de recém-nascidos prematuros para as faixas etárias de 28 a 31 semanas e 32 a 36 semanas. No grupo com maior número de observações (32 a 36 semanas), o percentual variou, no período de 2001 a 2005, de $83,2 \%$ a $83,6 \%$ $(\mathrm{p}<0,001)$.

Verificou-se também um alto percentual de valores ignorados para a variável idade gestacional em semanas nos anos de 1994, 1995 e 1996 $(19,7 \% ; 27 \%$ e $24,1 \%$, respectivamente). Isto se deveu, principalmente, a um grande número de valores ignorados para esta variável no Município de São Paulo. Quanto à evolução deste dado entre 1994 e 2005 (0,7\%), observa-se uma grande diminuição no número de nascimentos sem informação da idade gestacional $(p<0,001)$. Essa variação é menor se a comparação for feita com o ano de 1997 (de 3,7\% para 0,7\%), mas ainda estatisticamente significativa $(p<0,001)$.

A Figura 1 mostra a evolução da prematuridade na Região Norte, entre 1994 e 2005, segundo dados do SINASC. Observa-se uma diminuição dos nascimentos com menos de 37 semanas: de $7,2 \%$ dos nascidos vivos, em 1994 , para $5,1 \%$, em 2005 ( $\mathrm{p}<0,001)$. A proporção de valores ignorados para idade gestacional também diminuiu, de 2,5\%, em 1994, para 0,4\%, em 2005 ( $p<0,001)$. O percentual de partos realizados em hospitais na região vem se mantendo em torno dos $90 \%$ desde o ano de 1996.

Analisando as capitais da Região Norte, no mesmo período de 1994 a 2005, observa-se que o percentual de prematuridade apresentou aumento em Boa Vista (Roraima) (1,4\% para 3,8\%; p < 0,001), Macapá (Amapá) (3,7\% para 6,6\%; $\mathrm{p}<$ 0,001), Manaus (Amazonas) (3,8\% para $7 \%$; p < 0,001), Palmas (Tocantins) $(4,6 \%$ para $6,6 \% ; \mathrm{p}=$ $0,004)$, Porto Velho (Rondônia) (3,5\% para $5,8 \%$; p $<0,001)$ e Rio Branco (Acre) (2,4\% para 3,6\%; p < 0,001 ), e diminuição em Belém (Pará) (6,5\% para $5,7 \%$; $<<0,001)$. Ao se excluírem as capitais, a prematuridade na Região Norte, entre 1994 e 2005, diminuiu de 9,2\%, em 1994, para 4,7\%, em 2005. 
Percentual de partos prematuros (< 37 semanas) e de nascidos vivos com valores ignorados para a duração da gestação, segundo o Sistema de Informações sobre Nascidos Vivos (SINASC). Região Norte, Brasil, 1994 a 2005.

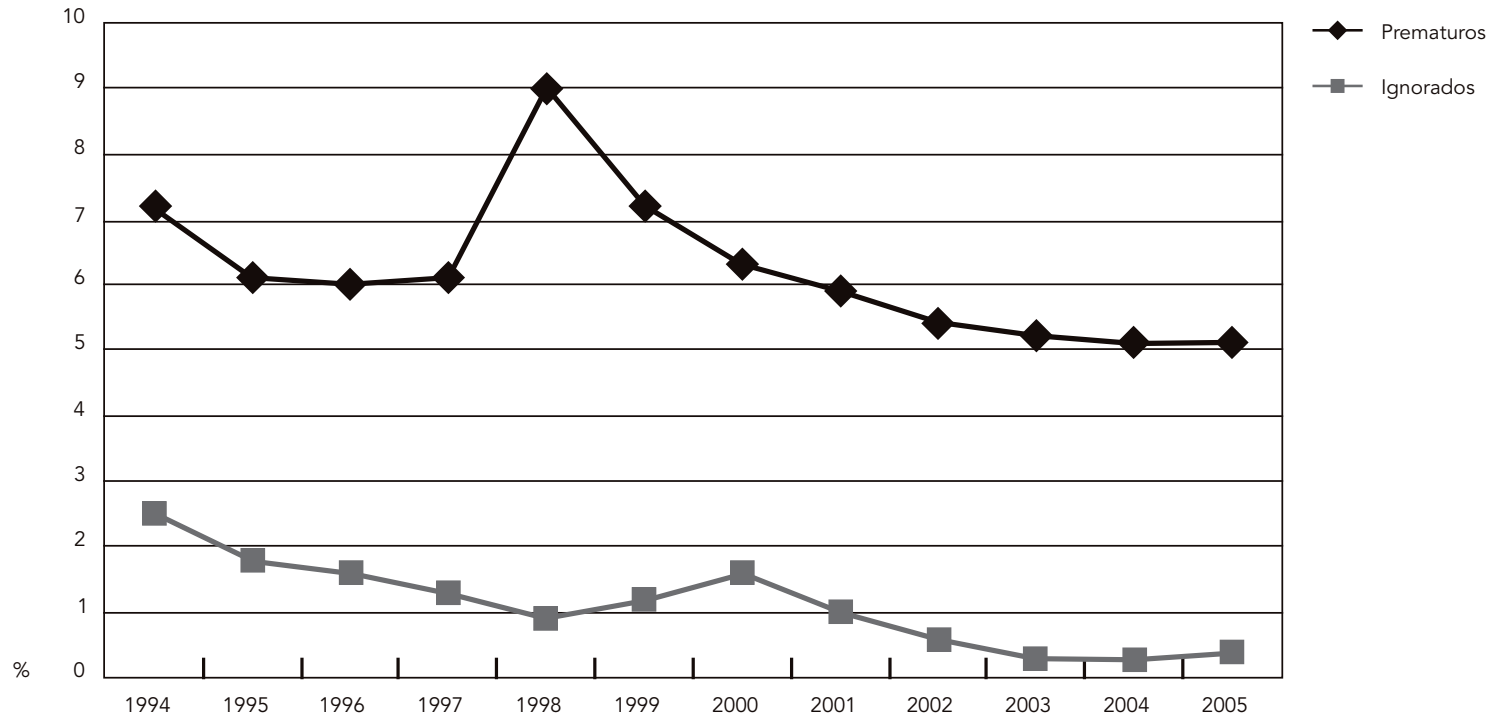

A Figura 2 apresenta os dados do SINASC para a Região Nordeste. Nessa região, no período estudado, observa-se diminuição dos nascimentos com menos de 37 semanas no início da série temporal (1994 a 1997) e posterior estabilização em torno de $6 \%$ a $5 \%$ em anos mais recentes (5,6\% dos nascidos vivos apresentaram prematuridade em 2005; $\mathrm{p}<0,001)$. A proporção de valores ignorados para idade gestacional diminuiu de forma consistente durante toda a série histórica, caindo de 8,9\%, em 1994, para 1\%, em 2005 (p $<0,001$ ). O percentual de partos hospitalares na Região Nordeste permaneceu acima de $90 \%$ nos anos estudados.

$\mathrm{Na}$ análise da evolução da prematuridade segundo o SINASC, nas capitais do Nordeste, observou-se para o período de 1994 a 2005 que o percentual de nascimentos com menos de 37 semanas aumentou em Aracaju (Sergipe) $(1,8 \%$ para 7,4\%; p < 0,001), João Pessoa (Paraíba) $(3,7 \%$ para $6,8 \%$; $<<0,001$ ), Natal (Rio Grande do Norte) $(4,1 \%$ para $7 \%$; $<0,001)$, Recife (Pernambuco) $(6,7 \%$ para $7,9 \%$; $<0,001)$, Salvador (Bahia) (5,6\% para 7,8\%; p < 0,001), São Luís (Maranhão) $(2,5 \%$ para $6,2 \%$; $<<0,001)$, Teresina (Piauí) $(3,7 \%$ para $8 \%$; $<<0,001$ ), e diminuiu em Fortaleza (Ceará) $(7,2 \%$ para $6,4 \% ; \mathrm{p}<0,001)$ e Maceió (Alagoas) ( $8 \%$ para $5,9 \%$; $<<0,001)$. Na análise da Região Nordeste, excluindo as capitais, verificou- se redução da prematuridade de 7,7\%, em 1994, para 5,2\%, em 2005.

A Figura 3 descreve a notificação ao SINASC de partos prematuros na Região Sudeste. Observou-se um aumento do percentual de partos prematuros, de $3,4 \%$, em 1994 , para $7,4 \%$, em 2005 ( $p<0,001$ ), e uma diminuição de valores ignorados de 39,5\%, em 1994, para 0,7\%, em 2005 ( $\mathrm{p}<0,001)$. Nessa região, o alto número de valores ignorados para a idade gestacional de 1994 a 1996 deve-se, principalmente, ao Município de São Paulo. Excluídos os dados deste município, verifica-se a persistência do aumento do percentual de partos prematuros $(3,7 \%$ para $7,2 \%$; p < 0,001). Se considerado apenas o período após 1996, quando o percentual de valores ignorados em São Paulo apresentou grande diminuição, observa-se ainda aumento da prematuridade $(5,4 \%$ para $7,4 \%$; $<<0,001)$ e diminuição no percentual de valores ignorados $(4,3 \%$ para $0,7 \% ; \mathrm{p}<0,001)$. O percentual de partos hospitalares nesta região permaneceu próximo a $100 \%$ durante o período estudado.

Na observação da evolução da prematuridade nas capitais do Sudeste, verificou-se, no período de 1994 a 2005, aumento do percentual de nascidos vivos prematuros em Belo Horizonte (Minas Gerais) $(5,8 \%$ para $9 \%$; $p<0,001)$ e Rio de Janeiro $(6,1 \%$ para $8,3 \% ; p<0,001)$. Também em São 
Figura 2

Percentual de partos prematuros e de nascidos vivos com valores ignorados para a duração da gestação, segundo o Sistema de Informações sobre Nascidos Vivos (SINASC). Região Nordeste, Brasil, 1994 a 2005.

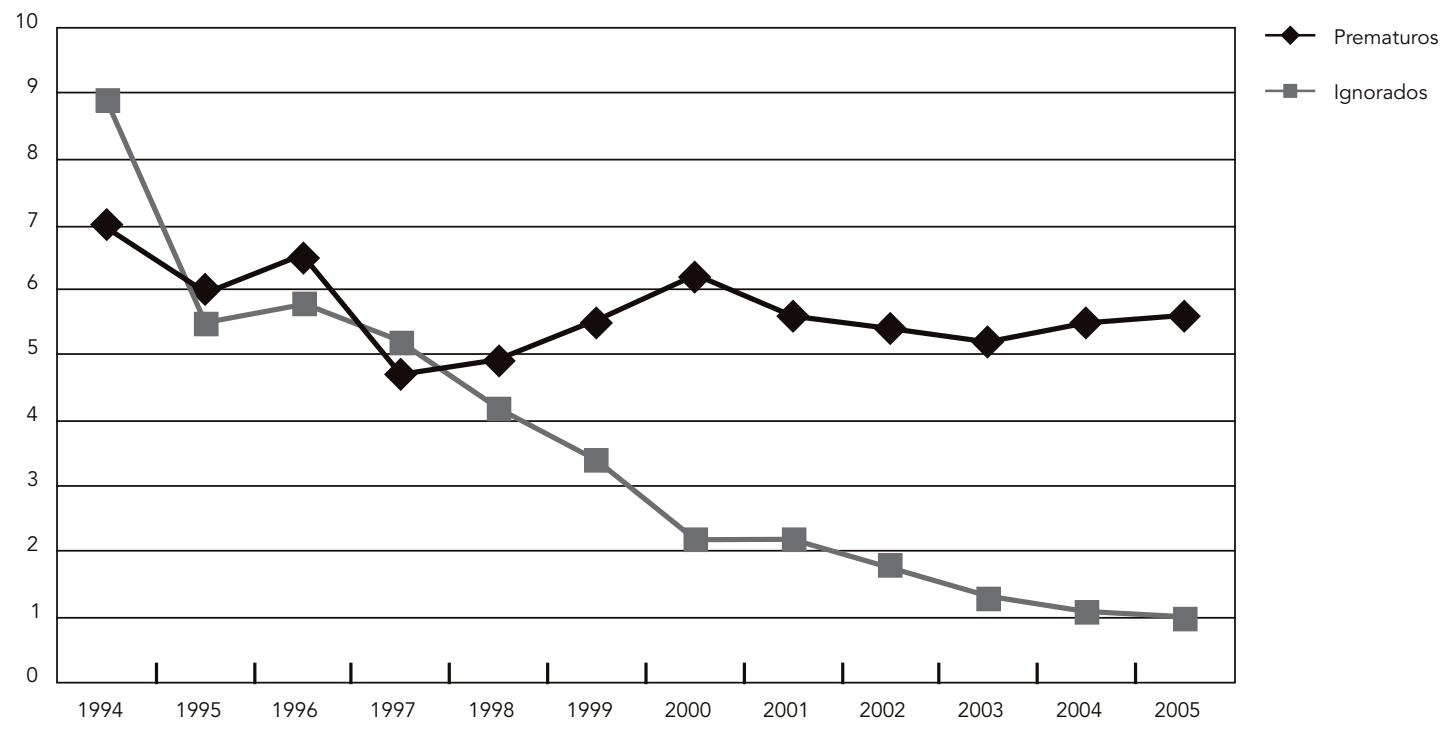

Figura 3

Percentual de partos prematuros e de nascidos vivos com valores ignorados para a duração da gestação, segundo Sistema de Informações sobre Nascidos Vivos (SINASC). Região Sudeste, Brasil, 1994 a 2005.

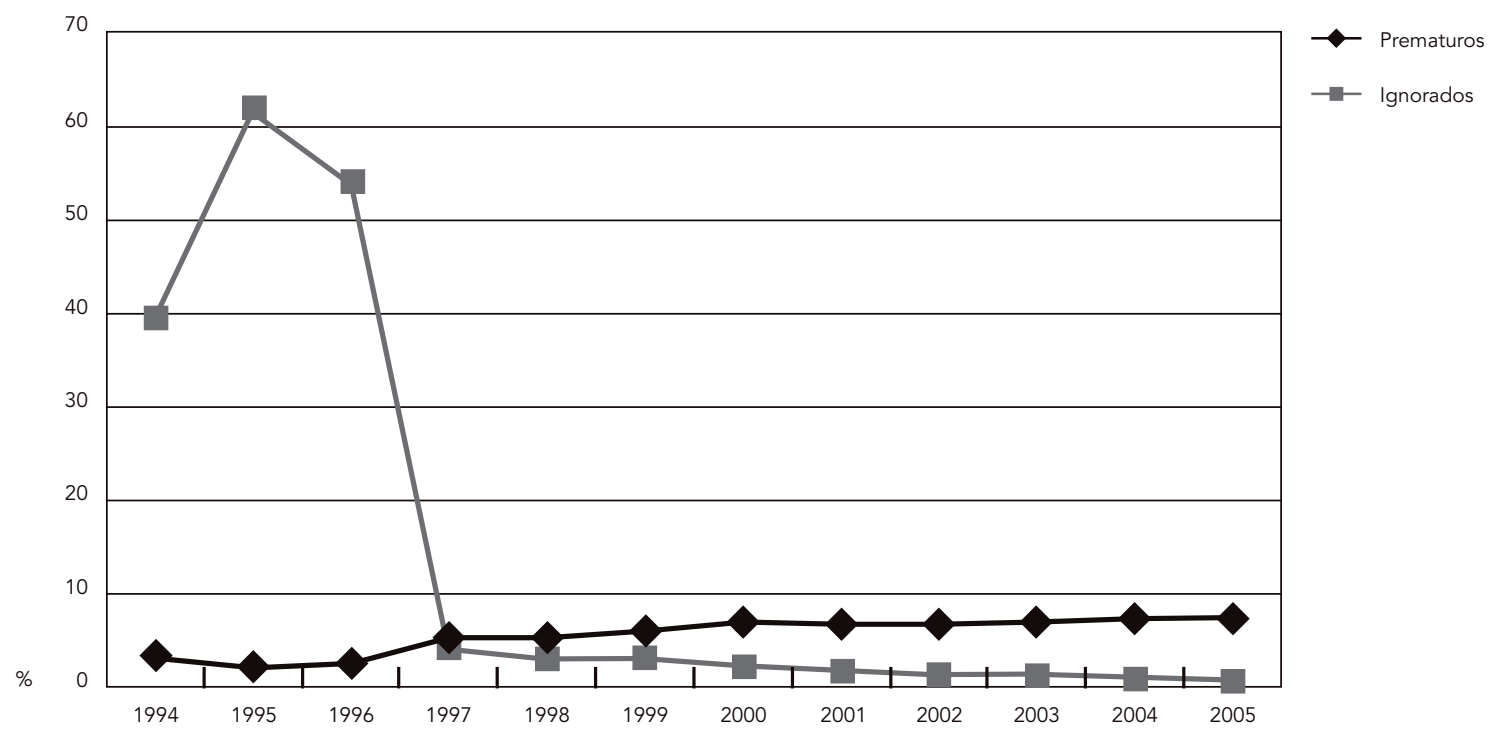


Paulo, no período de 1997 a 2005, observou-se aumento da prematuridade de $6,1 \%$ para $8,6 \%$ (p $<0,001$ ). Diminuição desse indicador foi observada apenas em Vitória (Espírito Santo) (9,6\% para $7,6 \% ; \mathrm{p}<0,001)$. Excluídas da análise as capitais, a prematuridade na Região Sudeste aumentou de $3,4 \%$ para $8,9 \%$ no período estudado.

Na Região Sul (Figura 4), o percentual de partos com duração menor de 37 semanas aumentou de 5,4\%, em 1994, para 7,3\%, em 2005 (p < $0,001)$, enquanto o percentual de valores ignorados para a duração da gestação diminuiu de 2,7\% para $0,2 \%(\mathrm{p}<0,001)$. O percentual de partos hospitalares esteve próximo a $100 \%$ neste período de análise. Ocorreu aumento do percentual de prematuros em todas as capitais da Região Sul entre 1994 e 2005: Curitiba (Paraná) (4,1\% para 6,2\%; $\mathrm{p}<0,001)$, Florianópolis (Santa Catarina) $(5,1 \%$ para 7,5\%; $<<0,001$ ) e Porto Alegre (Rio Grande do Sul) $(7,5 \%$ para $10,3 \%$; $<<0,001)$. Ao se excluírem as capitais da Região Sul, a prematuridade aumentou de $5,3 \%$ para $7,2 \%$.

A Figura 5 descreve os achados da Região Centro-Oeste. O percentual de partos prematuros variou de 4,2\%, em 1994, para 6,6\%, em 2005 ( $p<0,001)$, e o de valores ignorados, de $5,4 \%$ a $0,6 \%(\mathrm{p}<0,001)$. O percentual de partos hospitalares foi de $100 \%$ neste período de análise. Nas capitais desta região ocorreu aumento na ocorrência de prematuridade: em Brasília (Distrito Federal) (5\% para 9,7\%; p < 0,001), em Goiânia (Goiás) (3,2\% para 4,3\%; p < 0,001), em Cuiabá (Mato Grosso) (3,5\% para 7,4\%; p < 0,001) e em Campo Grande (Mato Grosso do Sul) (de 7\% para $10,6 \% ; \mathrm{p}<0,001)$. Ao se excluirem as capitais, a prematuridade na região aumentou de $3,8 \%$ para $5,5 \%$.

\section{Discussão}

O resultado da análise dos dados do SINASC indica que, no período de 1994 e 2005, ocorreu um aumento na taxa de prematuridade no Brasil em geral, partindo de 5\%, em 1994, para 5,4\%, em 1998, 5,6\%, em 2000, atingindo 6,6\%, em 2005. Existem ainda diferenças regionais importantes: em 2005, a prevalência de prematuridade foi de $7,4 \%$ na Região Sudeste e $5,1 \%$ na Região Norte. Este achado é consistente com artigo de revisão de estudos brasileiros de base populacional que identificou uma tendência de aumento da prematuridade ao longo dos anos 7 .

O número de dados ignorados para a variável "duração da idade gestacional" em semanas decresceu de forma significativa no mesmo

Figura 4

Percentual de partos prematuros e de nascidos vivos com valores ignorados para a duração da gestação, segundo o Sistema de Informaçães sobre Nascidos Vivos (SINASC). Região Sul, Brasil, 1994 a 2005.

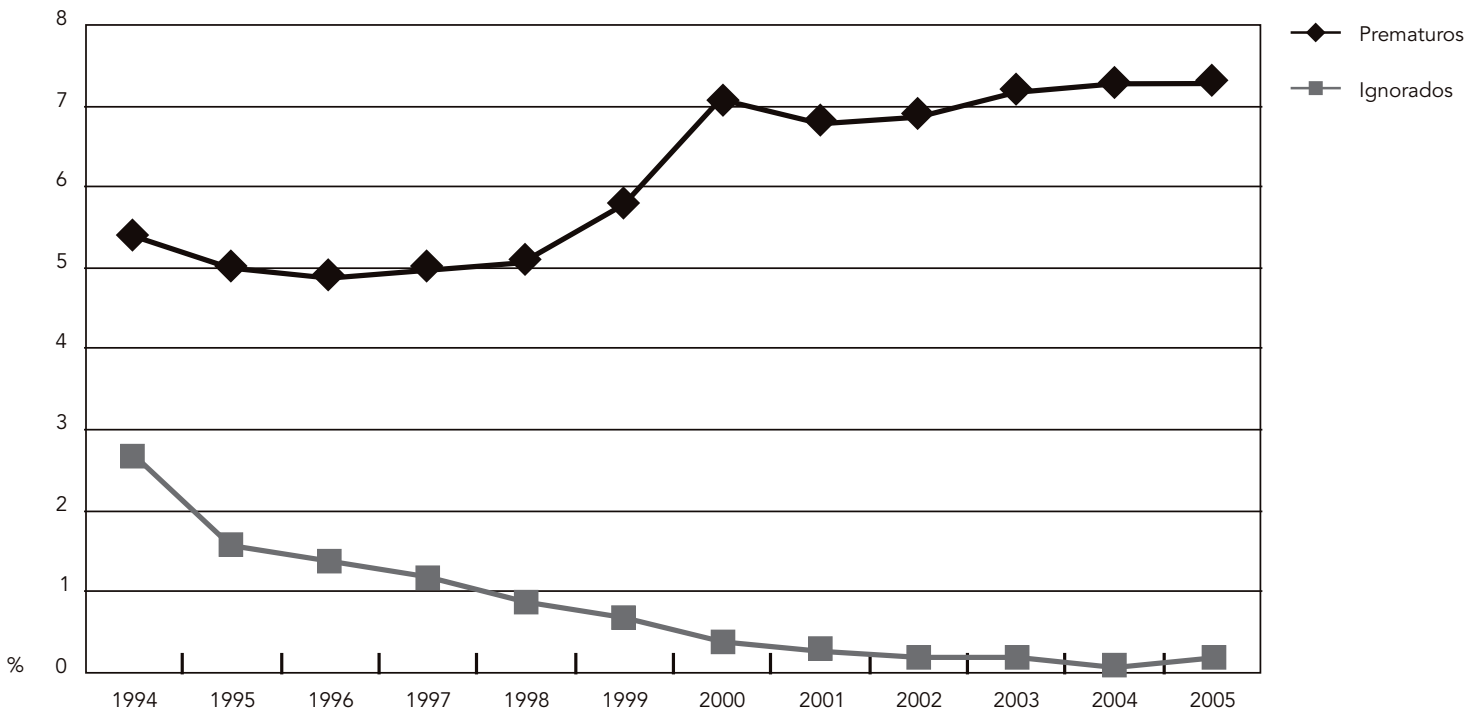




\section{Figura 5}

Percentual de partos prematuros e de nascidos vivos com valores ignorados para a duração da gestação, segundo o Sistema de Informações sobre Nascidos Vivos (SINASC). Região Centro-Oeste, Brasil, 1994 a 2005.

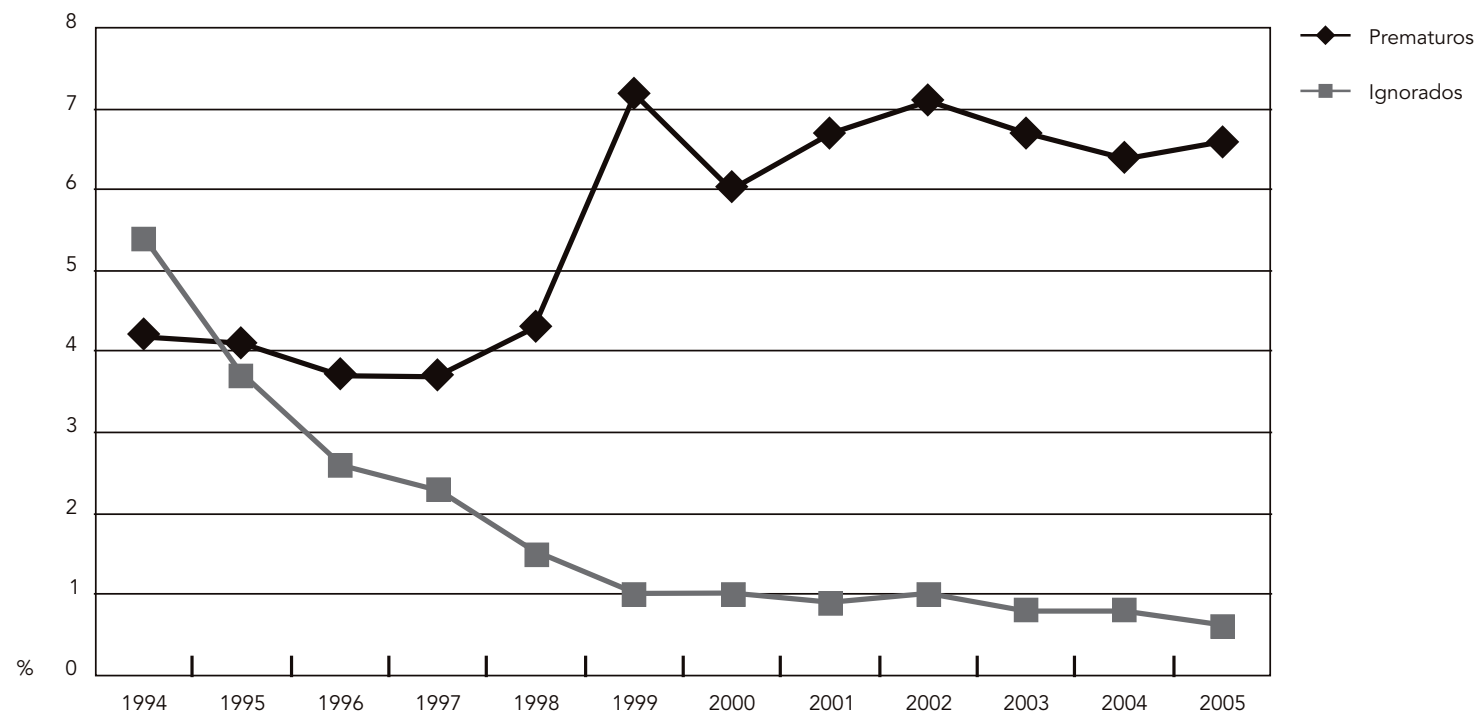

período, passando de 19,7\%, em 1994, para 3,7\%, em 1997, atingindo apenas 0,7\% em 2005. Um dos fatores que contribuiu para a redução expressiva desse indicador entre 1994 e 1997 foi a mudança ocorrida na metodologia de coleta de dados de nascidos vivos da Fundação Sistema Estadual de Análise de Dados (Fundação SEADE) de São Paulo, que, a partir de 1997, passou a coletar, sistematicamente, essa variável "duração da idade gestacional”. Por ser São Paulo o estado mais populoso do Brasil, a queda dos dados ignorados para essa variável foi expressiva no primeiro período da série analisada. A redução contínua nos anos seguintes demonstra a melhora progressiva no preenchimento das DNV e na digitação dos dados do SINASC. A queda de valores ignorados não é sinônimo de melhora na qualidade da informação, mas é uma condição necessária.

Pelos dados do SINASC, a taxa de prematuridade nas regiões Norte e Nordeste, entre 1994 e 2005, diminuiu, embora os nascimentos prétermo tenham aumentado na maior parte das capitais. As possíveis causas para essa redução vão desde a piora do preenchimento da DNV no que diz respeito à informação da idade gestacional, até a incorporação preferencial e enviesada no sistema de uma população de menor risco. Essa diminuição também pode estar associada a maior acesso aos serviços de saúde e melhoria na qualidade de vida, em anos recentes, no interior dos estados do Norte e do Nordeste. As causas diretamente associadas, no entanto, precisam ser mais bem estudadas. A diferença em relação às capitais pode ser explicada pelo fato de essas cidades concentrarem maternidades de alto risco e, por isso, contabilizarem maior número de nascimentos prematuros, que poderiam estar sendo notificados com locais de residência incorretos, ou ainda pela melhoria gradativa na qualidade do preenchimento da DNV. As regiões Sudeste, Sul e Centro-Oeste acompanharam a tendência do país, com aumento da taxa de prematuridade.

$\mathrm{Na}$ Tabela 1, podem-se observar as tendências de outros indicadores fornecidos pelo SINASC (peso ao nascer e tipo de parto), em uma tentativa de verificar a consistência dos dados de prematuridade desse sistema. Observa-se que, na Região Norte, a diminuição da prematuridade não é acompanhada pela diminuição do baixo peso ao nascer, sugerindo que os dados de idade gestacional sejam menos confiáveis. Na Região Nordeste, houve uma tendência de diminuição da prematuridade e do baixo peso ao nascer. Igualmente, no Sudeste e Sul, as tendências de aumento são consistentes entre estes dois indicadores, sugerindo melhor qualidade dos dados. 
No Centro-Oeste, houve tendência de aumento da prematuridade e do baixo peso ao nascer.

Em Pelotas (Região Sul), entre os anos de 1982 e 2004, a proporção de cesáreas aumentou significativamente, assim como a prematuridade, ocorrendo também um aumento na proporção de crianças com baixo peso ao nascer (mais por aumento dos partos pré-termo do que por restrição do crescimento intra-uterino). No entanto, relação direta entre esses dois processos não foi estabelecida, visto que o aumento dos partos pré-termo e a redução no peso médio ao nascer entre os anos de 1982 e 2004 afetou tanto os partos vaginais como os cesáreos 1 .

Em uma comparação entre duas coortes realizadas na década de 90, em São Luís (Região Nordeste) e Ribeirão Preto (São Paulo, Região Sudeste), encontrou-se menor taxa de baixo peso ao nascer na primeira, embora esta represente uma região menos desenvolvida do país. Por outro lado, as taxas de prematuridade entre essas duas cidades foram semelhantes. Os autores concluem que intervenções médicas mais precoces (cesárea e parto induzido) explicariam o maior número de nascidos vivos com baixo peso ao nascer em Ribeirão Preto 8 .

O SINASC vem apresentando progressiva melhora na cobertura e qualidade dos dados nos diversos estados e municípios brasileiros, havendo perspectivas de cobertura integral nos próximos anos 9 . A cobertura desse sistema para o Brasil como um todo, avaliada pela razão entre o número de nascidos vivos observados pelo SINASC e o número esperado, estimado pelo IBGE, variou de $85,5 \%$, em 1996 , para $89,3 \%$, em 2004 . No mesmo período, na Região Norte, este número passou de $70,4 \%$ para $90,8 \%$; no Nordeste, de $69,8 \%$ para $82,4 \%$; no Sudeste, de $93,4 \%$ para $91,9 \%$; no Sul, de 103,8\% para 96,2\%; no Centro-Oeste, de $103,3 \%$ para $93,6 \% 10$. A diminuição aparente no percentual de cobertura do SINASC nas regiões Sul e Sudeste, em relação às estimativas do IBGE, parece dever-se a uma maior sensibilidade do sistema para capturar mudanças importantes na dinâmica populacional (queda de fecundidade e natalidade) em anos recentes 6 .

A redução da natalidade ocorrida no Brasil reflete-se de maneira consistente na diminuição do número de nascidos vivos observada a partir de 1999 no SINASC e no número de registros de nascimentos dos cartórios de registro civil e no Sistema de Informação Hospitalar (SIH), fenômeno não completamente apreendido pelas técnicas de estimativas adotadas pelo IBGE 10. Esta concordância entre as três primeiras fontes agrega validade ao fenômeno de redução de nascimentos observado no SINASC, tornando remota a hipótese de deterioração ou redução de
Tabela 1

Variação da ocorrência de prematuridade e baixo peso ao nascer, nas regiões do Brasil, conforme o Sistema de Informações sobre Nascidos Vivos (SINASC), 1994 a 2005.

\begin{tabular}{lcc}
\hline Região & Partos & Baixo peso \\
\hline Norte & $-0,18$ & 2,65 \\
Nordeste & $-0,06$ & $-6,44$ \\
Sudeste & 0,42 & 1,16 \\
Sul & 0,26 & 8,31 \\
Centro-Oeste & 0,33 & 2,30 \\
\hline
\end{tabular}

cobertura desse sistema de informação no Brasil, menos ainda nas regiões Sul e Sudeste 10 .

A melhora da qualidade dos dados do SINASC tem estimulado seu uso para o gerenciamento das ações de saúde no país. A Rede Interagencial de Informações para a Saúde (RIPSA) tem utilizado o SINASC como fonte de dados para o denominador do cálculo da mortalidade infantil em vários estados brasileiros 6. O Ministério da Saúde, por sua vez, vem adotando as informações do SINASC como fonte de dados para o denominador do cálculo de cobertura vacinal desde 2005, na maioria dos estados, em face da dificuldade de utilizar as estimativas de nascidos nos anos intercensitários 10 .

Entretanto, ainda são notados problemas com a acurácia da idade gestacional originada do SINASC. Estudos brasileiros que investigaram a confiabilidade da idade gestacional fornecida pelo SINASC por meio de comparação com dados coletados em pesquisas encontraram valores de índice kappa variando de 0,09 a 0,83, com uma proporção de valores ignorados da ordem de $10 \%$ a $12,4 \% 11,12$.

Em estudo de casos e controles realizado por Almeida et al. 13, em São Paulo, no período de agosto de 2000 a janeiro de 2001, os autores observaram, entre os casos (óbitos neonatais precoces), uma pequena diferença $(2,9 \%)$ entre a prevalência considerada como real, retirada dos prontuários hospitalares, e a estimada de prematuridade com base no SINASC. Já entre os controles (crianças sobreviventes), a prevalência real de prematuridade foi de $8,4 \%$ e a estimada com base nos dados do SINASC, de 5,8\%, indicando que esse sistema subestimava em $30,8 \%$ a ocorrência de prematuridade 13 .

Quando comparados estudos de base populacional com dados do SINASC do mesmo local e ano, encontra-se grande disparidade na prevalência de prematuridade. A coorte de 1994 de Ribeirão Preto 14 mostrou uma prevalência de 
$13 \%$ de prematuridade, enquanto os dados do SINASC indicaram 4\%. O mesmo ocorreu em São Luís em 1997 e 1998, com 14\% pelo estudo de Silva et al. 15 e $2 \%$ pelo SINASC, e na coorte de 2004 de Pelotas, com 15\% pelo estudo de Barros et al. 16 e $10 \%$ pelo SINASC.

Uma das possíveis causas das grandes diferenças observadas inclui a qualidade da informação da idade gestacional, em virtude da forma de preenchimento da variável na DNV: categorizada e com largos intervalos na classificação da prematuridade (por exemplo, de 28 a 31 semanas, de 32 a 36 semanas e de 37 a 41 semanas de gestação). Esses intervalos podem induzir a erros de classificação das equipes locais, com tendência a migrar para faixas de idade gestacional mais próximas do termo. Dessa forma, seriam considerados prematuros somente os nascidos com idades mais extremas, sendo subnotificados os prematuros limítrofes. A prevalência de prematuridade tende a ser subestimada principalmente por causa de erros de classificação de nascidos vivos pré-termo com idade gestacional entre 34 e 36 semanas, classificados como de termo 11 . O registro da idade gestacional é, igualmente, menos padronizado

\section{Resumo}

A monitorização da prematuridade é muito importante, considerando seu impacto na morbidade e mortalidade infantis e seus custos econômicos e sociais. Este estudo utilizou dados do Sistema de Informações sobre Nascidos Vivos (SINASC), implantado em 1990 e expandido de forma gradativa até cobrir cerca de $90 \%$ de todos os nascimentos no país, para descrever a evolução da prematuridade no Brasil, regiões e capitais, entre os anos de 1994 e 2005. Observou-se um aumento na proporção de partos prematuros no país como um todo e uma diminuição no número de nascimentos sem informação da idade gestacional. As regiões Sudeste, Sul e Centro-Oeste acompanharam a tendência do país, ao contrário das regiões Norte e Nordeste. São comparados os achados do SINASC com os de estudos de base populacional. O SINASC vem apresentando progressiva melhora na cobertura e qualidade dos dados, mas ainda existem problemas com a acurácia da idade gestacional, levando a uma subestimação da prevalência de prematuridade. Por causa de sua importância, torna-se necessário aprofundar os esforços para o ganho de acurácia do sistema.

Prematuro; Sistemas de Informação; Planejamento em Saúde nos dados do SINASC, no qual também é apresentado maior número de valores ignorados do que nos dados de pesquisas.

Em virtude da importância do SINASC como ferramenta para avaliar a saúde perinatal em geral e, especialmente, a prevalência de prematuridade, torna-se necessário aprofundar os esforços para o ganho de acurácia do sistema. Nesse sentido, o Ministério da Saúde implantou, em 2004, os Comitês Técnicos Assessores (CTA) do Sistema de Informação sobre Mortalidade (SIM) e do SINASC, por meio da Portaria no. 16 17; além disso, instituiu um grupo-tarefa junto aos estados das regiões Norte e Nordeste, que visava a coletar subsídios para o aprimoramento do sistema. Entre as modificações sugeridas, está a forma de anotação da idade gestacional na DNV, que, a partir de 2008, passará a ser em número de semanas e não mais em intervalos, contribuindo para a melhora do registro.

Concomitantemente, será necessário promover a sensibilização dos profissionais para o correto preenchimento desse campo, de forma a possibilitar o adequado monitoramento da prematuridade em nosso país por meio das bases de dados coletados de rotina.

\section{Colaboradores}

M. F. Silveira participou da análise e redação do artigo. A. Matijasevich colaborou na redação do artigo e elaboração dos gráficos. I. S. Santos, D. C. Malta e E. C. Duarte contribuíram na redação do artigo.

\section{Agradecimentos}

Ao Fundo Nacional de Saúde, Ministério da Saúde (convênio MS/FNS 833/2006) pelo financiamento da pesquisa. 


\section{Referências}

1. Barros FC, Victora CG, Barros AJD, Santos IS, Albernaz E, Matijasevich A, et al. The challenge of reducing neonatal mortality in middle-income countries: findings from three Brazilian birth cohorts in 1982, 1993, and 2004. Lancet 2005; 365:847-54.

2. Davidoff MJ, Dias T, Damus K, Russell R, Bettegowda VR, Dolan S, et al. Changes in the gestational age distribution among U.S. singleton births: impact on rates of late preterm birth, 1992 to 2002 . Semin Perinatol 2006; 30:8-15.

3. Detels R, Holland WW, McEwen J, Omenn GS. Oxford textbook of public health. 3rd Ed. Oxford: Oxford University Press; 1997.

4. Murray CJL, Salomon JA, Mathers CD, Lopez AD. Summary measures of population health: concepts, ethics, measurement and applications. Geneva: World Health Organization; 2002.

5. Fundação Nacional de Saúde, Ministério da Saúde. Manual de procedimentos do Sistema de Informações sobre Nascidos Vivos. Brasília: Fundação Nacional de Saúde, Ministério da Saúde; 2001.

6. Departamento de Análise de Situação de Saúde, Secretaria de Vigilância em Saúde, Ministério da Saúde. Vigilância em saúde: dados e indicadores selecionados. Brasília: Ministério da Saúde; 2004.

7. Silveira MF, Santos IS, Barros AJD, Matijasevich A, Barros FC, Victora CG. Aumento da prematuridade no Brasil: revisão de estudos de base populacional. Rev Saúde Pública 2008; 42:957-64.

8. Silva AAM, Bettiol H, Barbieri MA, Ribeiro VS, Aragão VMF, Brito LGO, et al. Infant mortality and low birth weight in cities of Northeastern and Southeastern Brazil. Rev Saúde Pública 2003; 37:693-8.

9. Departamento de Análise da Situação de Saúde, Secretaria de Vigilância em Saúde, Ministério da Saúde. Saúde Brasil 2006 - uma análise da situação de saúde. Brasília: Ministério da Saúde; 2006.
10. Departamento de Análise da Situação de Saúde, Secretaria de Vigilância em Saúde, Ministério da Saúde. Saúde Brasil 2004 - uma análise da situação de saúde. Brasília: Ministério da Saúde; 2004.

11. Silva AAM, Ribeiro VS, Borba Júnior AF, Coimbra LC, Silva RA. Avaliação da qualidade dos dados do Sistema de Informações sobre Nascidos Vivos em 1997-1998. Rev Saúde Pública 2001; 35:508-14.

12. Theme Filha MM, Gama SGN, Cunha CB, Leal MC. Confiabilidade do Sistema de Informações sobre Nascidos Vivos hospitalares no Município do Rio de Janeiro, 1999-2001. Cad Saúde Pública 2004; 20:583-91.

13. Almeida MF, Alencar GP, França Jr. I, Novaes HMD, Siqueira AAF, Schoeps D, et al. Validade das informações das declarações de nascidos vivos com base em estudo de caso-controle. Cad Saúde Pública 2006; 22:643-52.

14. Bettiol H, Rona RJ, Chinn S, Goldani M, Barbieri MA. Factors associated with preterm births in Southeast Brazil: a comparison of two birth cohorts born 15 years apart. Paediatr Perinat Epidemiol 2000; 14:30-8.

15. Silva AAM, Coimbra LC, Silva RA, Alves MTSSB, Lamy Filho F, Lamy ZC, et al. Perinatal health and mother-child health care in the municipality of São Luís, Maranhão State, Brazil. Cad Saúde Pública 2001; 17:1413-23.

16. Barros AJD, Santos IS, Victora CG, Albernaz EP, Domingues MR, Timm IK, et al. Coorte de nascimentos de Pelotas, 2004: metodologia e descrição. Rev Saúde Pública 2006; 40:402-13.

17. Ministério da Saúde. Portaria no ${ }^{\circ}$. 16. Institui o Comitê Técnico Assessor do SINASC. Diário Oficial da União 2004; 26 abr.

Recebido em 25/Abr/2008

Versão final reapresentada em 06/Nov/2008

Aprovado em 09/Fev/2009 